\title{
Ga-assisted growth of GaAs nanowires on silicon, comparison of surface SiOx of different nature
}

\author{
Federico Matteini, Gözde Tütüncüoğlu, Daniel Rüffer, Esther Alarcón-Lladó, \\ Anna Fontcuberta i Morral* \\ Laboratoire des Matériaux Semiconducteurs, École Polytechnique Fédérale de Lausanne, EPFL STI IMX LMSC (MXC) Station 12, \\ CH-1015 Lausanne, Switzerland
}

\section{A R T I C L E I N F O}

\section{Article history:}

Received 23 June 2014

Received in revised form

18 July 2014

Accepted 19 July 2014

Communicated by K. Deppert

Available online 29 July 2014

Keywords:

A1. Nanostructures

A3. Molecular beam epitaxy

B1. Nanomaterials

B2. Semiconducting gallium arsenide

\begin{abstract}
A B S T R A C T
Physical properties of surfaces are extremely important for initiation and nucleation of crystal growth, including nanowires. In recent years, fluctuations in surface characteristics have often been related to unreproducible growth of GaAs nanowires on Si by the Ga-assisted method. We report on a systematic study of the occurrence of GaAs nanowire growth on silicon by the Ga-assisted method for different kinds of silicon oxides: native, thermal and hydrogen silsesquioxane (HSQ). We find that success in achieving nanowires and the growth conditions such as gallium rate and substrate temperature depend mainly on the physical properties of the surface: oxide stoichiometry, oxide thickness and surface roughness. These results constitute a step further towards the integration of GaAs technology on the Si platform.
\end{abstract}

c) 2014 Elsevier B.V. All rights reserved.

\section{Introduction}

Semiconducting nanowires provide a wide range of possibilities both in applied and fundamental science [1-3]. Within the range of possible applications III-V semiconductors are among the most promising materials. The small footprint of nanowires allows for virtually defect-free integration of mismatched materials, which would not be possible in the thin film form [4,5]. Moreover, III-V nanowires can be obtained on Si [6-11], providing a path for combining the III-V and Si platforms. As nanowires start growing generally in a single nucleation event followed by a layer-by-layer mode, III-V nanowires grown on silicon appear also free from antiphase boundaries otherwise often found in thin film counterparts [12-14]. Still, the lack of polarity of the silicon substrate leads to non-perpendicular growth nanowires grown on $\mathrm{Si}(111)$ due to the existence of three-dimensional twinning under certain growth conditions $[13,15]$.

One of the most successful ways of growing nanowires is the vapor-liquid-solid method (VLS) in which a liquid droplet (denominated as catalyst) is used for the gathering and preferential decomposition of growth precursors $[16,17]$. Upon supersaturation of the droplet, precipitation occurs at the interface with the substrate in the form of a nanowire. One of the most successful catalysts used for VLS is gold. However, when heating a Si substrate

\footnotetext{
*Corresponding author. Tel.: +41 2169 37394; fax: +41 216937368.

E-mail address: anna.fontcuberta-morral@epfl.ch (A. Fontcuberta i Morral).
}

with gold on top, the gold droplets incorporate a significant amount of silicon by the formation of an eutectic alloy. The presence of $\mathrm{Si}$ in the $\mathrm{Au}$ interferes with the decomposition and precipitation of precursors. As a consequence, growth of III-V nanowires on $\mathrm{Si}$ using gold as a catalyst is quite more challenging than on III-Vs. Many groups working on the growth of III-V nanowires on silicon have looked for alternative methods, including the selective area epitaxy and Ga-assisted growth of GaAs nanowires [6-8,13,18-21]. In the latter, it was found that the presence of an oxide at the surface was necessary for the formation of the liquid droplet necessary for VLS.

The physical characteristics of the silicon oxide have always been an important parameter in Ga-assisted nanowire growth [9-11]. It was observed that the oxide thickness plays a role in achieving nanowires with an epitaxial relation to the substrate [22]. Interestingly, the critical thicknesses reported by the various groups are significantly different depending on the preparation method of the oxide: $5 \mathrm{~nm}$ for Hydrogen Silsesquioxane (HSQ), $0.9-2 \mathrm{~nm}$ for thermal oxide and $30 \mathrm{~nm}$ for sputtered oxide [22,23]. In these works, the existence of a critical oxide thickness on a GaAs substrate was discussed in terms of the opening of craters in the oxide, either by the reaction of Ga with the substoichiometric oxide and/or due to the desorption of As at GaAs surface temperatures above $500{ }^{\circ} \mathrm{C}$. Few works report on successful growth without the presence of an oxide on the silicon surface $[24,7,10]$. One should note that in all of these cases, there was a nonnegligible time lapse between substrate preparation and loading in the ultra-high-vacuum environment. It is well established that 
Si surfaces naturally undergo oxidation even at room temperature, simply by exposing them to air. The same oxidation process takes place also in the case of hydrogen passivated surfaces [25-27]. As a consequence, what was claimed as oxide-free surface had most probably oxidized to $\mathrm{Si}+1$ (i.e. $\mathrm{Si}-\mathrm{O}-\mathrm{Si}$ ) as shown from X-Ray photoelectron spectroscopy data of refs $[10,28]$.

Most of the works discussing the role of the oxide in the growth of GaAs nanowires by the Ga-assisted method were performed on GaAs substrates [22,7,9-11]. To the best of our knowledge, there are no reports on the role of oxide in Ga-assisted growth of GaAs nanowires on silicon. Different groups have used different types of surface preparation, but no systematic comparison between different types of oxide has been realized. Additionally, it is generally accepted that growth conditions for obtaining GaAs nanowires can strongly depend on the wafer batches and providers used, despite identical nominal properties. There is a clear need for finding out the physical origin of this in order to deduce a reproducible preparation method of the silicon surface. In this work, we address the following unanswered questions on the Ga-assisted growth of GaAs nanowires on silicon: is the presence of an oxide needed? What is the ideal oxide stoichiometry for reproducible growth? What is the ideal critical oxide thickness? Is it possible to relate the physical characteristics of the surface with the ideal growth conditions? For this, we analyze the physical characteristics of different types of oxides and relate them to the optimized GaAs nanowires growth conditions. This work constitutes a step towards systematic use of silicon as a substrate for Ga-assisted growth of GaAs nanowires and could be potentially extended to other materials systems.

\section{Experimental details}

GaAs nanowires have been grown by Ga-assisted self-catalyzed method on Si(111) 2-inch wafers RCA treated (ended with an HF etch) from different providers (Virginia and Siltronix, p-doped Boron, $10-20 \Omega \mathrm{cm}$ ) from now on named Batch A and B respectively. The growth was performed in a Molecular Beam Epitaxy machine (MBE) with solid state sources (DCA P600). Previous to growth and in order to ensure a clean surface, all substrates were annealed at $500{ }^{\circ} \mathrm{C}$ in a separate UHV chamber for $2 \mathrm{~h}$; from now on we will refer to this process as degassing. The effect of this step on oxide chemistry, thickness and roughness is reported in the Supplementary Information. All the values of roughness, thickness and chemistry characterization reported in the manuscript have been performed after degassing, unless differently specified. After this step, the samples were moved to the growth chamber, always in UHV.

The silicon substrates were prepared with different types of oxides: thermal, native and Hydrogen Silsesquioxane (HSQ). Thermal oxide was produced by means of dry oxidation of the wafer of batch B in a Centrotherm furnace at $950{ }^{\circ} \mathrm{C}$ in a cleanroom environment. The native oxide was obtained by natural exposure of the Si wafers (of batch A and B) to air in cleanroom environment $\left(21 \pm 0.5^{\circ} \mathrm{C}\right.$, humidity $\left.44 \%\right)$. HSQ oxide was obtained by spinning a HSQ:MIBK solution (XR-1541-002, Dow Corning) on wafers of batch $\mathrm{B}$ at $6000 \mathrm{rpm}$ with subsequent annealing for $5 \mathrm{~min}$ at $180{ }^{\circ} \mathrm{C}$ for removal of the solvent. Without diluting the solution, the oxide thickness achieved was of 28-30 nm. By diluting it (1:4$1: 8)$, thinner oxides were obtained $(8-4 \mathrm{~nm})$. The films were transformed into silicon oxide by annealing them at $475{ }^{\circ} \mathrm{C}$ in $\mathrm{N}_{2}$ atmosphere for $1 \mathrm{~h}$. The solutions were spun right after exposing the Si wafers to an HF solution, in order to avoid the presence of the native oxide. The oxide thickness was controllably reduced by chemical etching with a $\mathrm{NH}_{4} \mathrm{~F}: \mathrm{HF}(500: 1)$ solution. The etching rate was calibrated independently for every type of oxide used.
The oxide thickness was measured with spectroscopic ellipsometry (Sopra GES 5E) and confirmed by Atomic Force Microscopy (AFM) on etched steps. Each thickness value obtained by ellipsometry is the result of the average of five measurements in different points on the wafer. Attenuated total reflection (ATR) IR spectroscopy (Jasco FT/IR 6300 with Pike MIRacle holder with single reflection diamond crystal) was realized for the characterization of the oxide stoichiometry, by scanning in the $650-4000 \mathrm{~cm}^{-1}$ range with 100 accumulations. Although, since the intensity of the signal-to-noise ratio above $1500 \mathrm{~cm}^{-1}$ is extremely low, only the low range $\left(650-1500 \mathrm{~cm}^{-1}\right)$ is considered and reported. Each ATR-FTIR measurement was repeated 3 times on each sample in different points. Finally, AFM (Bruker) was also used for the determination of the surface roughness: each surface roughness value is the result of the average of three measurements of $5 \times 5 \mu \mathrm{m}, 1 \times 1 \mu \mathrm{m}$ and $500 \times 500 \mathrm{~nm}$ areas. It is important to remark that the reported values of roughness have been measured after the degassing. The roughness before degassing are reported in the Supplementary Information. In the case of completely etched oxides, the substrates were immersed in an isopropanol bath immediately after etching, and then dried under Nitrogen flow just before loading in the UHV environment. We used the following range of growth conditions:

- the substrate temperature between $580{ }^{\circ} \mathrm{C}$ and $660{ }^{\circ} \mathrm{C}$, as measured by means of a calibrated pyrometer;

- Ga rates between $0.25 \mathrm{~A} / \mathrm{s}$ and $1.25 \mathrm{~A} / \mathrm{s}$, as calibrated on planar growth on GaAs (111) by means of Reflection High Energy Electron Diffraction (RHEED);

- $\mathrm{As}_{4}$ fluxes were from $2.5 \times 10^{-6}$ torr to $4.9 \times 10^{-6}$ torr; as calibrated by means of a beam flux monitor gauge.

Scanning Electron Microscopy (SEM) (Zeiss Merlin) was used for the morphological characterization of the samples.

\section{Experimental results}

\subsection{Chemical composition of the oxides}

We start by presenting the nature of the various oxides used in this work. Thermal oxide is a mostly stoichiometric oxide $\left(\mathrm{SiO}_{2}\right)$, which can be produced by oxidation of Silicon at $800-1200{ }^{\circ} \mathrm{C}$ under a controlled oxygen flux; it exhibits low as-grown roughness $(\sim>0.6 \mathrm{~nm})[34]$.

Native oxide is a thin layer of oxide formed by the natural exposure of a Si wafer to air; it follows the surface roughness of the underlying silicon substrate and it grows monolayer by monolayer $[25,35,27]$. The chemical composition of native oxide depends on its thickness. For thicknesses of few monolayers, it mainly consists of $\mathrm{Si}-\mathrm{O}-\mathrm{Si}$ [36]. The oxygen content increases for larger thicknesses, though it remains sub-stoichiometric with respect to thermal oxide.

HSQ oxide is obtained by annealing a Hydrogen Silsesquioxane resin on a silicon wafer previously etched with HF. The thickness can be tuned by the dilution of the resin solution and the spinning rate $[37,38]$. Annealing the $\mathrm{HSQ}$ resin at $450{ }^{\circ} \mathrm{C}$ transforms the cage structure of HSQ monomer into a network, whose chemical composition is $\mathrm{SiO}_{x}$ with $1<x<2$, depending on the annealing temperature $[37,38]$.

By investigating these three types of oxides, we can understand the influence of the stoichiometry in the nucleation and growth mechanism of GaAs nanowires. The stoichiometry of silicon oxide can be characterized with Fourier Transform Infrared Spectroscopy (FTIR). The main absorption bands characteristic of silicon oxide are the interstitial oxygen band $(\mathrm{Si}-\mathrm{O}-\mathrm{Si})$, centered at $1107 \mathrm{~cm}^{-1}$, 
$[29,31,32]$ the transverse optical phonon (TO) of $\mathrm{SiO}_{2}$ around $1075 \mathrm{~cm}^{-1}$ [33], as well as the longitudinal optical phonon (LO) around $1250 \mathrm{~cm}^{-1}$ [33], as reported in Table 1 . Suboxides of the form $\mathrm{SiO}_{x}$ with $1 \leq x<2$ are characterized by an absorption band

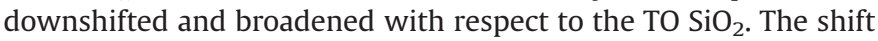
can be directly related to the stoichiometry [33]. Examples of ATRFTIR spectra obtained from the different oxides are shown in Fig. 1.

In Fig. 1 the $\mathrm{Si}-\mathrm{O}-\mathrm{Si}$ absorption band is observed for all the oxides (thermal, native and HSQ), albeit with different intensities. The spectra at higher wavenumbers depend on the oxide type:

- Thermal oxide shows a clear $\mathrm{LO}-\mathrm{SiO}_{2}$ positioned at $1250 \mathrm{~cm}^{-1}$, indicating it being stoichiometric oxide.

- HSQ oxide shows an absorption band $\left(1226 \mathrm{~cm}^{-1}\right)$, indicating that it is substoichiometric oxide $\mathrm{SiO}_{x}$ with $(1 \leq x<2)$ [33].

- Native oxide does not show any additional absorption band than the already described Si-O-Si related, consistently with reported results on films of similar thickness [29].

Table 1

Characteristic phononic modes of silicon oxide.

\begin{tabular}{lcl}
\hline Phononic mode & Position $\left(\mathrm{cm}^{-1}\right)$ & Ref. \\
\hline $\mathrm{Si}-\mathrm{O}-\mathrm{Si}$ & 1107 & {$[29-32]$} \\
$\mathrm{TO} \mathrm{SiO}_{2}$ & 1075 & {$[33]$} \\
$\mathrm{LO} \mathrm{SiO}_{2}$ & 1250 & {$[33]$} \\
\hline
\end{tabular}

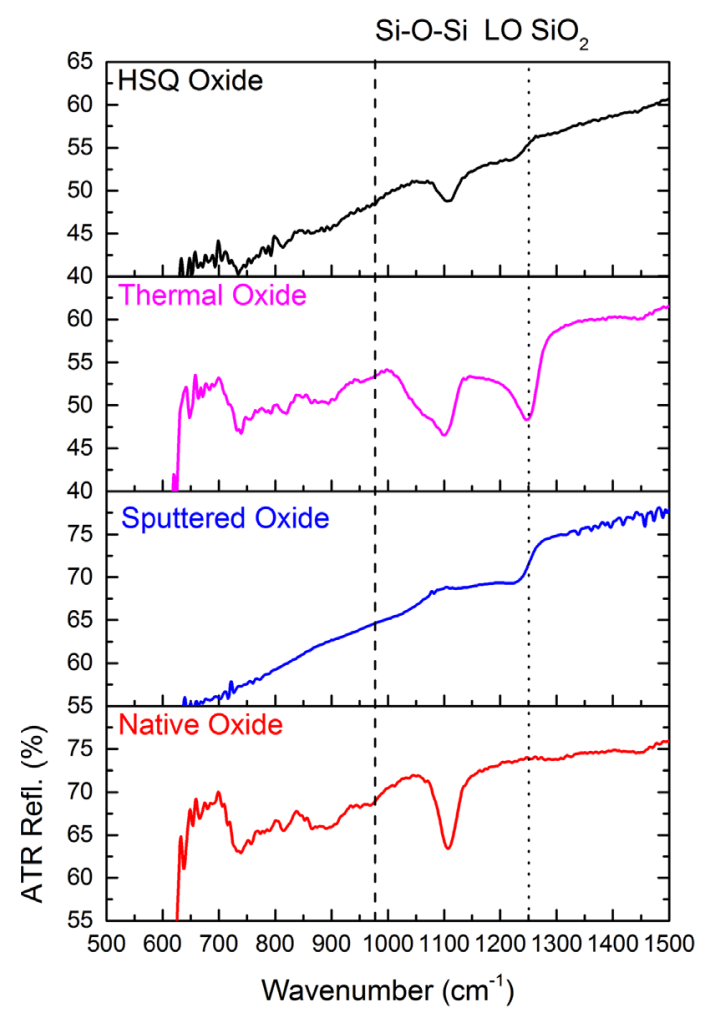

Fig. 1. ATR FTIR spectroscopy of different oxides. The peak at $1250 \mathrm{~cm}^{-1}$ corresponds to the LO mode of $\mathrm{SiO}_{2}$, whereas the peak at $1107 \mathrm{~cm}^{-1}$ is related to the presence of interstitial oxide $\mathrm{Si}-\mathrm{O}-\mathrm{Si}$. The $\mathrm{TO}$ mode of $\mathrm{SiO}_{2}$ is located around $1075 \mathrm{~cm}^{-1}$. Thermal oxide is the only oxide that shows TO and $\mathrm{LO}$ modes of $\mathrm{SiO}_{2}$. HSQ presents a downshifted broad peak around $1225 \mathrm{~cm}^{-1}$ correspondent to nonstoichiometric oxide $\mathrm{SiO}_{x}$ with $x<2$. All the oxides show the interstitial oxide peak $\left(1107 \mathrm{~cm}^{-1}\right)$ but not the sputtered oxide on GaAs; this proves that $\mathrm{Si}-\mathrm{O}-\mathrm{Si}$ is peculiar of $\mathrm{Si} / \mathrm{SiO}_{2}$-interface. A similar result was obtained with HSQ oxide on GaAs substrate (see Supplementary Information).
In order to demonstrate that the interstitial oxide is characteristic only of the interface between the silicon and the silicon oxide, the spectra of sputtered oxide on GaAs is also shown (see Fig. 1); the same result has been achieved with HSQ on GaAs (see Supplementary Information). No absorption band is observed at $1107 \mathrm{~cm}^{-1}$, showing that no interstitial oxide is present. On the other hand a broad absorption is observed around $1226 \mathrm{~cm}^{-1}$ characteristic of sub-stoichiometric oxide. As a remark, interstitial oxide is present in every type of oxides on silicon since it represent the initial step of oxidation of every silicon surface, being followed by oxide of different composition, depending on the processing [27]. In the following, FTIR spectrum of Si substrate is used as a tool to investigate the physical properties that influence the early stages of growth of self-catalyzed GaAs nanowires.

\subsection{Thermal oxide}

We first present our results on thermally oxidized silicon substrates. As introduced earlier in this manuscript, three physical properties of the substrates will be considered: thickness, roughness and chemical composition. First we investigate substrates with four different thicknesses of the same oxide, by a combination of optical lithography and etching (see supplementary information). This allowed us to investigate simultaneously several oxide thicknesses under identical experimental conditions.

Fig. 2 shows SEM micrographs of oxidized substrates with varying oxide thicknesses after performing the same growth process. The growth conditions were substrate temperature $T=600^{\circ} \mathrm{C}$, Gallium rate $\mathrm{Ga}=1.25 \mathrm{~A} / \mathrm{s}$ and Arsenic beam flux pressure $\mathrm{As}=2.5 \times$ $10^{-6}$ torr. Under these conditions nanowire growth was observed only for an oxide thickness between 1 and $2 \mathrm{~nm}$ (Fig. 2(c)). Similar thickness selectivity results were obtained under other conditions ( $T=590-630^{\circ} \mathrm{C}$, Gallium rate $0.5-1.25 \mathrm{~A} / \mathrm{s}$ ) leading to nanowire growth. In the case of thicker oxides, Ga droplets were always observed on the surface (see Fig. 2(a) and (b)). Oppositely, for oxidefree silicon surfaces, textured two-dimensional growth was found (see Fig. 2(d)). Such a change in growth mechanism could be related to the difference in surface energy of the $\mathrm{SiO}_{2}$ compared to the bare $\mathrm{Si}$ and thereby influence the initial Ga droplet formation and pinning on the surface. The question here is what makes $1-2 \mathrm{~nm}$ thermal oxide so prone for Ga-assisted nanowire growth. In order to shed some light to this question, we show the ATR-FTIR spectra of the thermal oxide of different thickness (see Fig. 2 (e)), with comparable pre-degassing roughness $(\sim 0.3 \mathrm{~nm})$. It is interesting to note that the intensity of the $\mathrm{LO} \mathrm{SiO}_{2}$ is proportional to the thickness, while the Si-O-Si mode exhibits the identical amplitude for all. This corroborates the interface nature of $\mathrm{Si}-\mathrm{O}-\mathrm{Si}$. Moreover, when the oxide thickness is around $1-2 \mathrm{~nm}$, roughness was comparable to thickness (see Table 2), and only the $\mathrm{Si}-\mathrm{O}-\mathrm{Si}$ band is observed. These results are in good agreement with the results obtained by Muller et al. [34]: stoichiometric silicon dioxide is formed only for thicknesses higher than $2 \mathrm{~nm}$.

\subsection{Native oxide}

From our study on thermal oxide we understand that when roughness is comparable to thickness, nanowire growth can be achieved. In other words, this condition corresponds to the exposure of $\mathrm{Si} / \mathrm{SiO}_{2}$-interface (i.e. $\mathrm{Si}-\mathrm{O}-\mathrm{Si}$ ) Based on the thermal oxide results, we also speculate that the substoichiometric composition of the oxide might help in the achievement of nanowire growth. As described in the first section, native oxide appears naturally upon exposing silicon wafers to air. This would supposedly make native oxide a reliable candidate. Still, one observes an extreme variation within batches and providers on the success in nanowire growth, which to date is not understood. In order to 

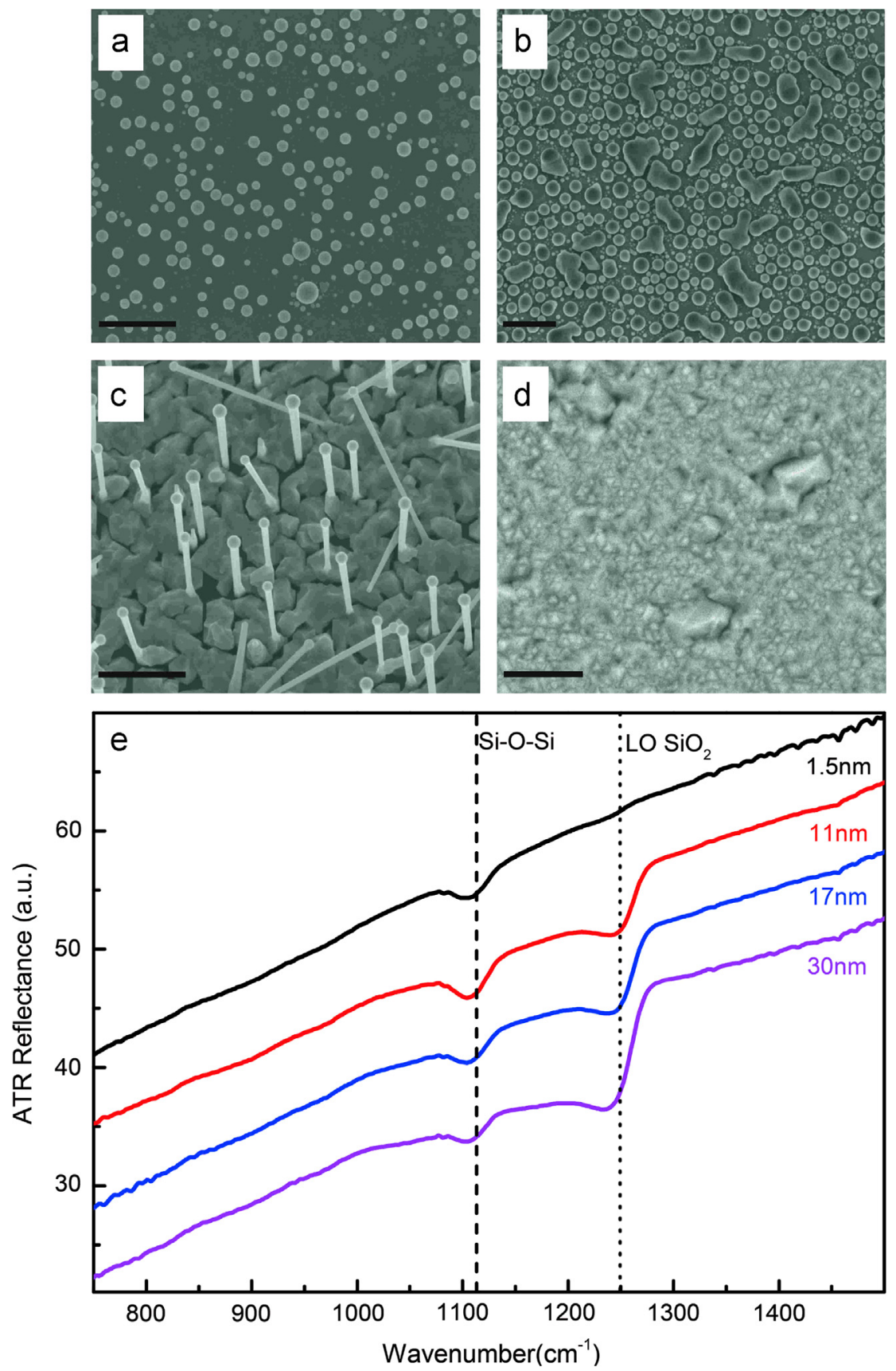

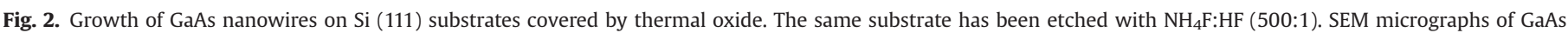

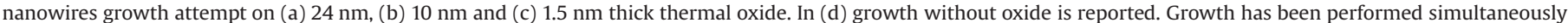

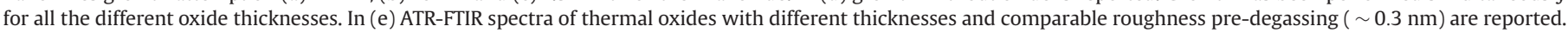
By decreasing the oxide thickness the $\mathrm{LO}-\mathrm{SiO}_{2}$ is decreasing in intensity, whereas the $\mathrm{Si}-\mathrm{O}-\mathrm{Si}$ remain unchanged. The scale-bar corresponds to $1 \mu \mathrm{m}$.

Table 2

Thickness and roughness of different types of oxides after degassing. The measurements have been performed by ellipsometry.

\begin{tabular}{llll}
\hline Oxide type & Annealing $\left({ }^{\circ} \mathrm{C}\right)$ & Thickness $(\mathrm{nm})$ & Roughness RMS $(\mathrm{nm})$ \\
\hline Native oxide (Batch A) & 500 & $0.8 \pm 0.6$ & $0.5 \pm 0.5$ \\
Native oxide (Batch B) & 500 & $2.1 \pm 0.6$ & $5.3 \pm 0.5$ \\
Thermal oxide & 500 & $1.2 \pm 0.6$ & $1.3 \pm 0.5$ \\
HSQ etched & 300 & $8.8 \pm 0.9$ & $3.1 \pm 0.5$ \\
HSQ as spun & 300 & $8.1 \pm 0.6$ & $1.2 \pm 0.5$ \\
\hline
\end{tabular}

demonstrate that this variability is not a provider issue, we take a closer look at the physical properties of the native oxide of substrates coming from different providers that, despite having identical specifications, gave rise to different growths. One should note that the characteristics may not be specific of a certain company and may vary from batch to batch. As a first observation we find that at comparable roughness pre-degassing $(0.3-0.8 \mathrm{~nm}$, see supplementary information) the oxide is slightly thicker in the case of batch B than batch A (see Table 2). Secondly, the ATR-FTIR spectra (Fig. 3 (a)) show that the intensity of the Si-O-Si peak is much higher for Batch $\mathrm{B}$ wafers, pointing out a rougher $\mathrm{Si} / \mathrm{SiO}_{2^{-}}$ interface. This is in accordance with the post degassing roughness measurements (see Table 2). Finally, we show the SEM micrographs of the growth performed on the two type of substrates (see Fig. 3 (b) and (c)). The two growths were performed under identical conditions $\left(T=610^{\circ} \mathrm{C}, \mathrm{Ga}=0.27 \mathrm{~A} / \mathrm{s}\right.$, As $=2.5 \times 10^{-6}$ torr $)$. However, while a forest of nanowires is obtained on batch $\mathrm{B}$ wafers 

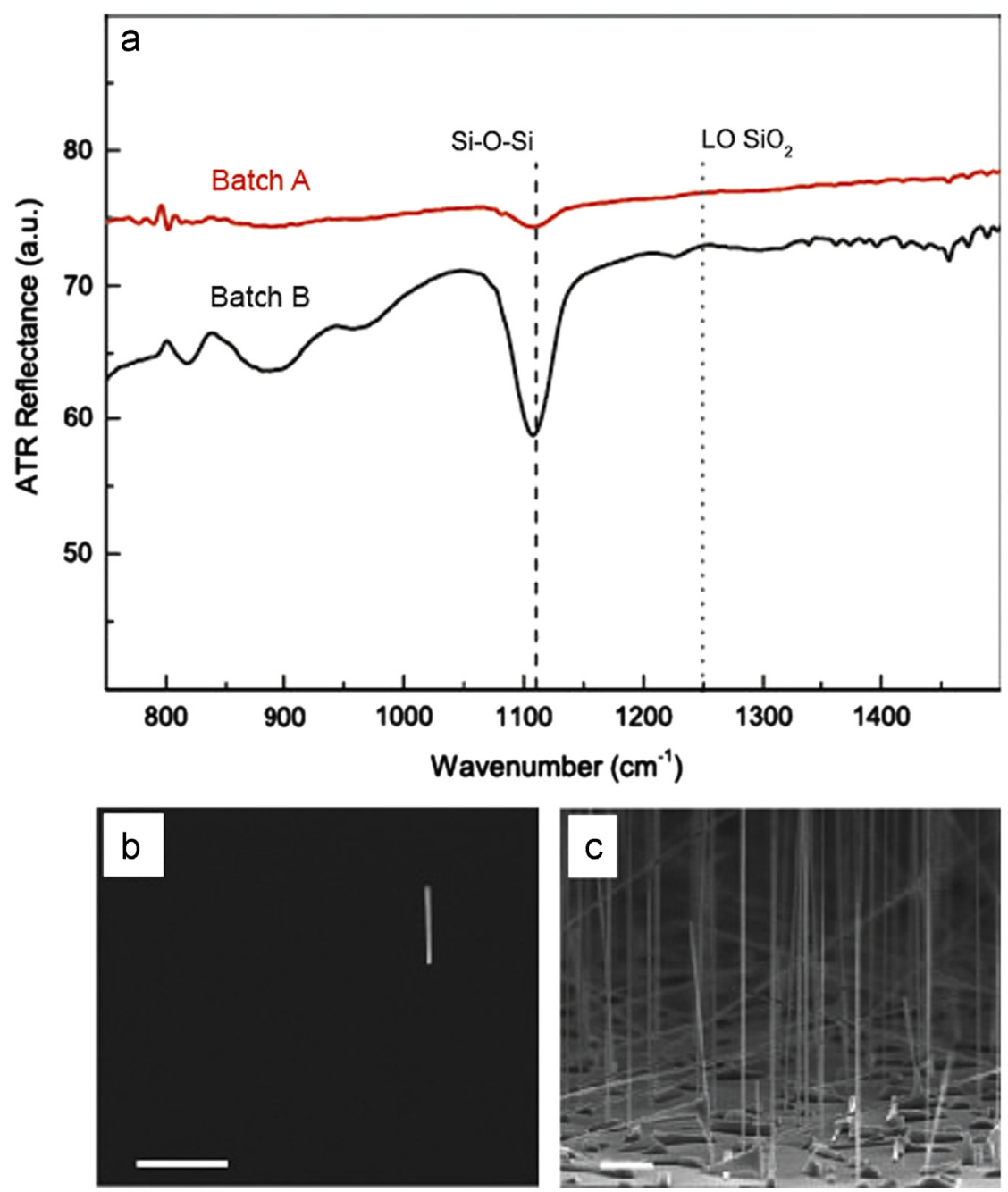

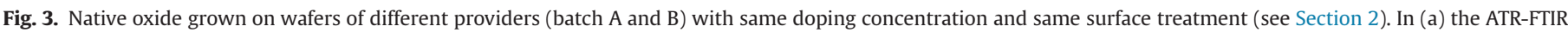

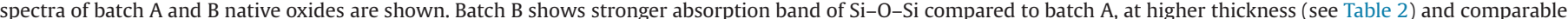

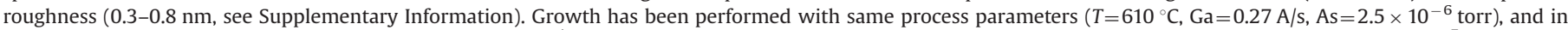

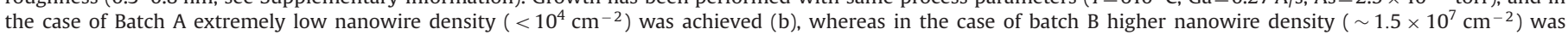
achieved (c). The scale-bar corresponds to $2 \mu \mathrm{m}$.

(roughest surface), an extremely low density of nanowires is observed on the batch A (smoothest surface). The different nanowire density can be understood in terms of changes in surface diffusion: a lower roughness results in an augmented surface diffusion, that lead to a lower nanowire density [39]. Vice versa, a rougher substrate (i.e. batch $\mathrm{B}$ ) leads to a higher nanowire density, as shown in Fig. 3 (c). In a nutshell, the $\mathrm{Si} / \mathrm{SiO}_{2}$-interface seems to be a key parameter in the GaAs nanowire growth on Si. Further studies should involve the intentional modification of this roughness for engineering the nanowire density in a reproducible manner.

\subsection{HSQ oxide}

So far we have evaluated the growth on $\mathrm{SiO}_{2}$ and interstitial oxide ( $\mathrm{Si}-\mathrm{O}-\mathrm{Si}$ ) exposed to the surface. Now we want to evaluate the possibility of growth on an oxide with intermediate composition. For this purpose we used silicon substrates covered with silicon oxide from HSQ processing. Further information about the processing of HSQ can be found in the Supplementary Information. Analogously to the thermal oxide investigation, several growths were performed simultaneously on oxide thicknesses ranging from $2 \mathrm{~nm}$ up to $24 \mathrm{~nm}$. Representative SEM images of samples grown under at a substrate temperature of $595^{\circ} \mathrm{C}$, Ga rate of $1.1 \mathrm{~A} / \mathrm{s}$ and As flux of $2.5 \times 10^{-6}$ torr are reported in Fig. 4. As the SEM micrographs show, growth of vertical nanowires was only achieved for thin oxide layers $(<5-6 \mathrm{~nm})$. This value is higher than in the case of thermal oxide, and comparable to what has been reported for HSQ on GaAs substrates [40]. Similar to what was observed for thermal oxide, the $\mathrm{Si}-\mathrm{O}-\mathrm{Si}$ band is the most dominant feature in the FTIR spectra of HSQ oxide of around the critical thickness and below. We note that the IR spectrum of $4 \mathrm{~nm}$ thick HSQ presents a non-negligible $\mathrm{SiO}_{x}$-related absorption band.

For this reason we performed the following experiment; we prepared two substrates with the same HSQ oxide thickness, achieved by

- Spinning HSQ from a MIBK diluted solution (1:8) to form directly a film with a thickness of $4-5 \mathrm{~nm}$. This type of sample will be called "as spun".

- Spinning HSQ from non-diluted solution, and etched it down to a similar thickness. In the following, this type of sample will be called "etched".

The IR spectra are virtually identical (see Fig. 5 (a)), indicating the same composition of $\mathrm{Si}-\mathrm{O}-\mathrm{Si}$ and $\mathrm{SiO}_{x}$. Interestingly, successful 

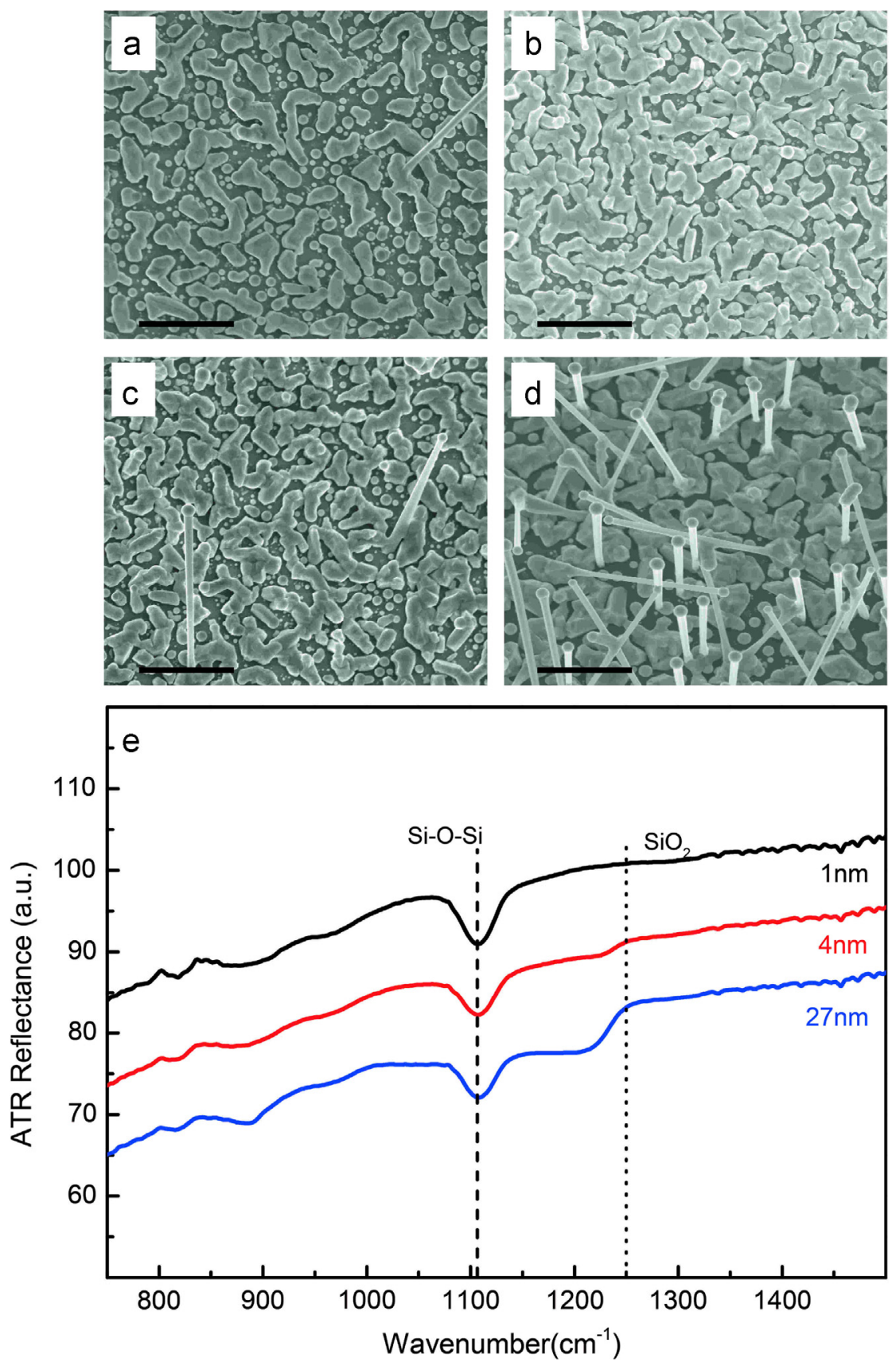

Fig. 4. Growth of GaAs nanowires on $\mathrm{Si}$ (111) substrates covered with HSQ oxide. Thickness was controlled by etching down the oxide with $\mathrm{NH}_{4} \mathrm{~F}: \mathrm{HF}$ diluted 500:1. In (a)-(b)(c) and (d) the SEM micrographs show the growth attempts on respectively 19-15-8 and $5 \mathrm{~nm}$ oxide thicknesses. Only in the latter case growth was performed successfully; the growth conditions were substrate temperature $595^{\circ} \mathrm{C}$, Ga rate of $1.1 \mathrm{~A} / \mathrm{s}$ and As flux of $2.5 \times 10^{-6}$ torr. (e) ATR-FTIR spectra of HSQ oxides with different thicknesses: the absorption band of $\mathrm{SiO}_{x}$ decreases in intensity by decreasing oxide thickness. On the other hand the absorption band of Si-O-Si does not decrease in intensity, showing that it is related to the $\mathrm{Si} / \mathrm{SiO}_{2}$-interface. The scale-bar corresponds to $2 \mu \mathrm{m}$.

growth had been achieved only in the case of etched HSQ (see Fig. 5). Even though the substrates seemed to be identical, the AFM analysis revealed a difference in surface roughness (see Table 2). In the case of low roughness (as spun) only Ga droplets were obtained, whereas for roughness of the order of the oxide thickness (etched), vertical nanowires were observed. We believe that, as schematically depicted in inset of Fig. 5, the Ga droplet cannot reach the $\mathrm{Si} / \mathrm{SiO}_{2}$-interface and no growth is observed in the case of a smooth and compact oxide layer. On the other hand, for large surface roughness (i.e. comparable to thickness), the Si/ $\mathrm{SiO}_{2}$-interface can be exposed to the precursors, allowing vertical nanowire growth.

\section{Discussion}

We discuss now the results in views of generalizing our findings for nanowire growth. We first review the impact of stoichiometry and roughness of the oxide on nanowire growth. We have varied the composition of the silicon oxide $\mathrm{SiO}_{x}$ with $x$ ranging from 0 to 2 . For simplicity's sake, we start considering growth on the two extreme cases: stoichiometric $\mathrm{SiO}_{2}(x=2)$, namely thermal oxide thicker than $2 \mathrm{~nm}$, and oxide-free silicon $(x=0)$, see for example Fig. 2 . In both cases it was not possible to obtain GaAs nanowire growth in any of the conditions used. Instead, we observe a droplet-like deposit on the $\mathrm{SiO}_{2}$, while a 

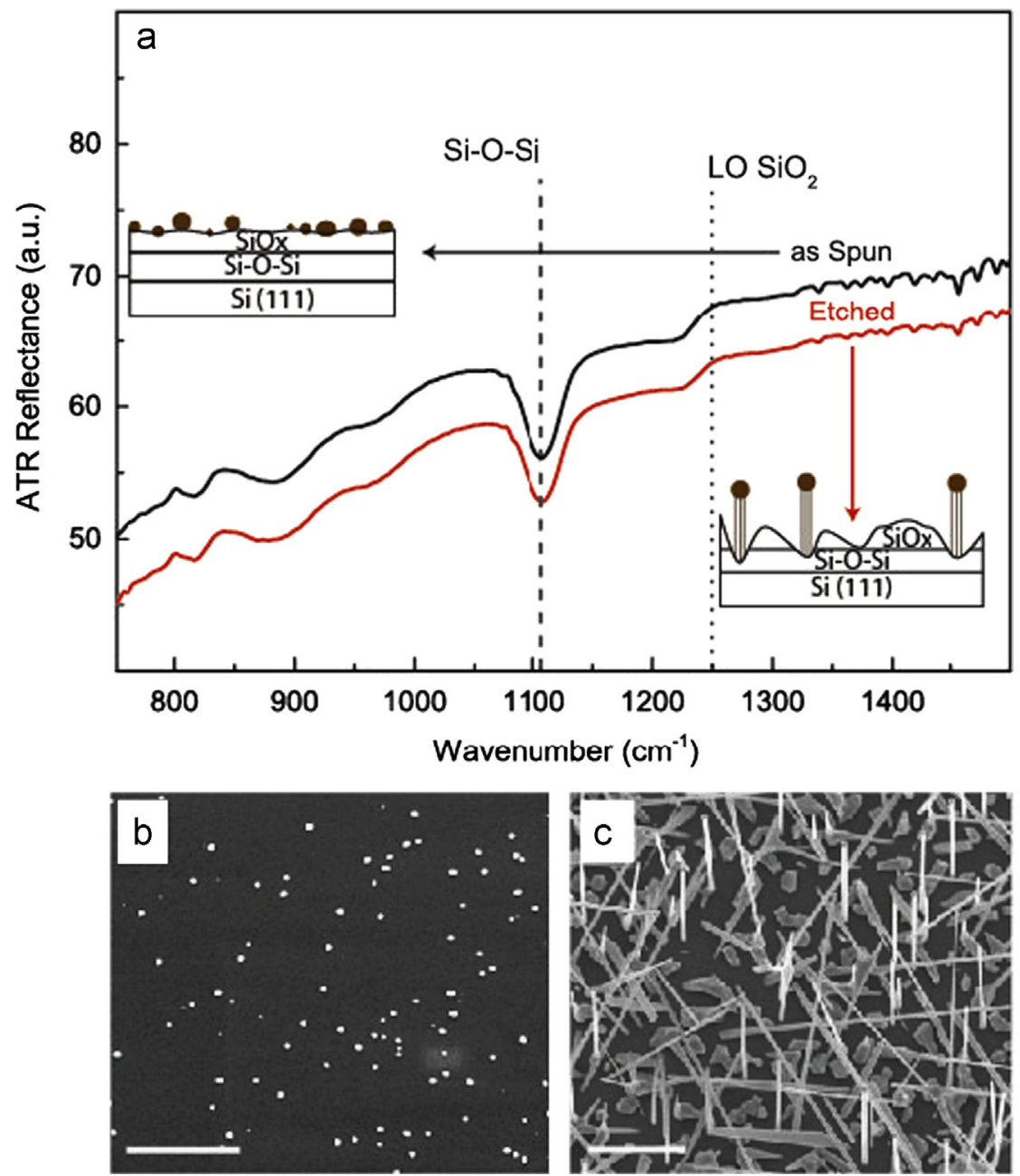

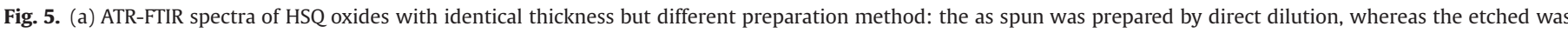

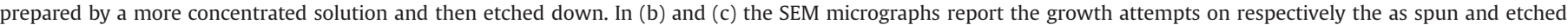

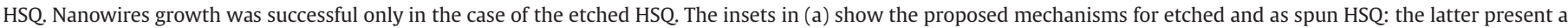

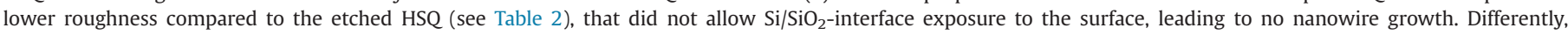

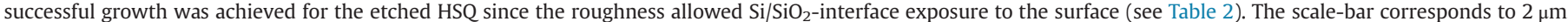

polycrystalline growth is observed in the oxide-free surface. The droplet-like deposit suggests that the diffusion coefficient of Ga-adatoms on the oxide is higher than on the silicon surface. It is interesting to note that in the case of growth on Si substrates covered with oxide thickness comparable to roughness, nanowires were always obtained. This condition suggests a nucleation model based on the formation of a pin-hole that provides the epitaxial relation with the substrate, coherently with what reported from other researchers $[41,22]$. The conditions of roughness comparable to thickness that allow the pin-hole formation, necessary to achieve nanowire growth can be obtained in various manners: thermal oxide thinner than $2 \mathrm{~nm}$, HSQ etched thinner than $4-5 \mathrm{~nm}$ and native oxide. A clear proof of the importance of roughness vs thickness is shown in Fig. 5 (b) and (c), where two substrates were prepared with HSQ with identical thickness and composition, but different roughness. We have also seen that the roughness/thickness conditions that lead to successful growth seems to be correlated to $\mathrm{Si} / \mathrm{SiO}_{2}$-interface (i.e. $\mathrm{Si}-\mathrm{O}-\mathrm{Si}$ ), as shown in Fig. 6. The recurrent observation of such an interface layer has been described in other works, where though successful growth of GaAs nanowires on patterned silicon dioxide is not related to the presence of interstitial oxide at the $\mathrm{SiO}_{2}$ hole opening [42-44]. However the role of $\mathrm{Si} / \mathrm{SiO}_{2}$-interface in the growth mechanism is still not fully understood and requires further investigation.

In parallel, we explored growth on smooth surfaces (batch A substrates) to understand if nanowire density could be controlled by the "processing parameters", as Ga rate or substrate temperature. We varied both Ga rate and substrate temperature respectively from $0.3 \mathrm{~A} / \mathrm{s}$ to $1.1 \mathrm{~A} / \mathrm{s}$, and from $600{ }^{\circ} \mathrm{C}$ to $660{ }^{\circ} \mathrm{C}$, as shown in Fig. 6. At low Ga flux and substrate temperatures ((a) and (b)) extremely low density of vertical nanowires is observed, as already reported in Fig. 3. On the other hand the higher the Ga flux ((c)(e)-(g)), the more material is deposited on the surface, resulting in an increased nanowire density and polycrystalline parasitic islands density. By increasing temperature, the polycrystalline parasitic layer decreases $((\mathrm{g})-(\mathrm{h})-(\mathrm{i}))$. Coherently with what mentioned before, the diffusion length of Ga atoms augment with increasing temperature, decreasing the polycrystalline islands density.

In order to generalize these results, we have correlated the surface characteristics with the conditions needed to achieve growth with comparable density number of vertical nanowires $\left((1.5 \pm 0.5) \times 10^{7} \mathrm{~cm}^{-2}\right)$. Fig. 7 depicts the general conditions for obtaining comparable nanowire density as a function of surface roughness and $\mathrm{Si}-\mathrm{O}-\mathrm{Si}$ content. In general, a lower surface roughness requires higher substrate temperature and Ga rate to achieve 


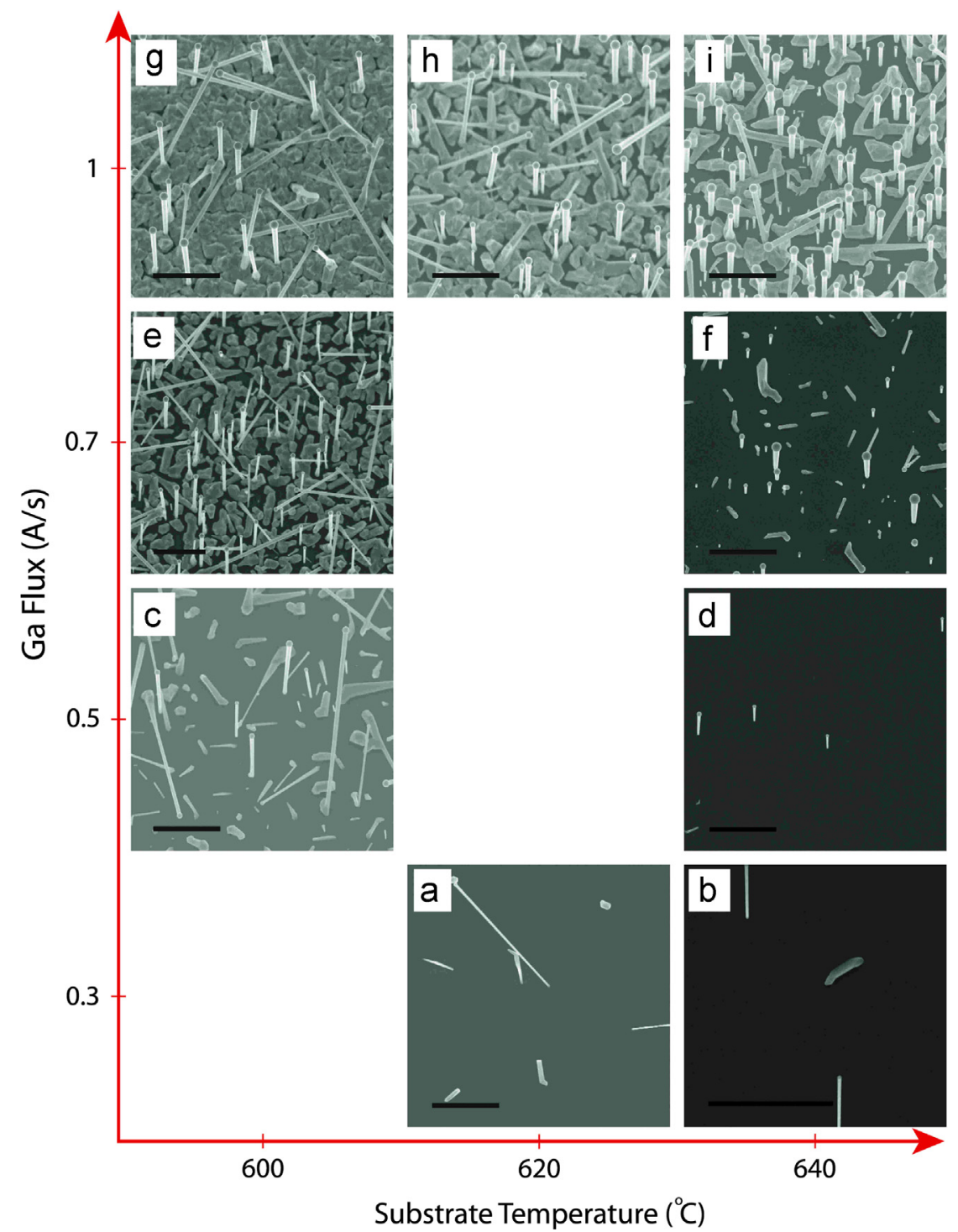

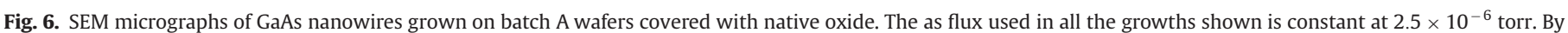

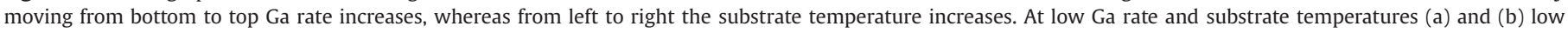

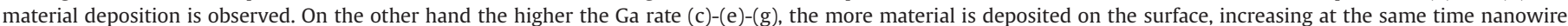

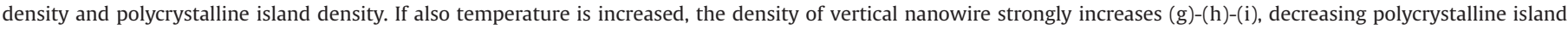
density. Growth at temperature above $640{ }^{\circ} \mathrm{C}$ was also attempted, but no growth was observed anymore. The scale-bar corresponds to $2 \mu \mathrm{m}$.

comparable nanowire density. As an example, in the case of batch A Si wafers (smoothest surface), the conditions to achieve the desired nanowire density were of $\mathrm{Ga}=0.75 \mathrm{~A} / \mathrm{s}$ and $T_{\text {sub }}=640{ }^{\circ} \mathrm{C}$.

Thermal oxide presented the second lowest surface roughness: the desired nanowire density was achieved with $\mathrm{Ga}=0.75 \mathrm{~A} / \mathrm{s}$ and $T_{\text {sub }}=625^{\circ} \mathrm{C}$. The trend is followed by HSQ for which the conditions for the desired nanowire density were $\mathrm{Ga}=0.45 \mathrm{~A} / \mathrm{s}$ and $T_{\text {sub }}=610^{\circ} \mathrm{C}$. In the case of the highest surface roughness, a comparable nanowire density was achieved at $\mathrm{Ga}=0.27 \mathrm{~A} / \mathrm{s}$ and $T_{\text {sub }}=610,{ }^{\circ} \mathrm{C}$.

We explain the change in Ga rate conditions for creating comparable nanowire density with surface diffusion: a decrease in roughness produce an increase in surface diffusion, forming less nanowires [39]. Therefore, to increase nanowire density for lower roughness substrates (i.e. native oxide batch A) Ga rate and substrate temperature have to be increased compared to rougher substrate (i.e. native oxide batch B).

As shown in Fig. 7, roughness and interstitial oxide content are directly correlated, suggesting that the sharpness of the
$\mathrm{Si} / \mathrm{SiO}_{2}$-interface affects the characteristic roughness for the bottom up oxides (i.e. thermal oxide and native oxide). Presently, how to achieve control over interstitial oxide formation remains still open and it needs further investigation.

\section{Conclusions}

In conclusion, we have shown that roughness is a key parameter for forming pinholes necessary for successful GaAs nanowire growth. This explains the different optimal oxide thicknesses to achieve growth reported in literature: the critical oxide thickness depends on the surface roughness as it is related to the thickness leaving $\mathrm{Si} / \mathrm{SiO}_{2}$-interface exposed to the surface. For example, we found a critical thickness of 1-2 nm for thermal and native oxide, 5-6 nm for oxide from HSQ. The lower the roughness, the higher the temperature and the Ga rate needed for achieving the comparable nanowire density, and vice versa. Additionally, we have also shown that the "provider dependence" 


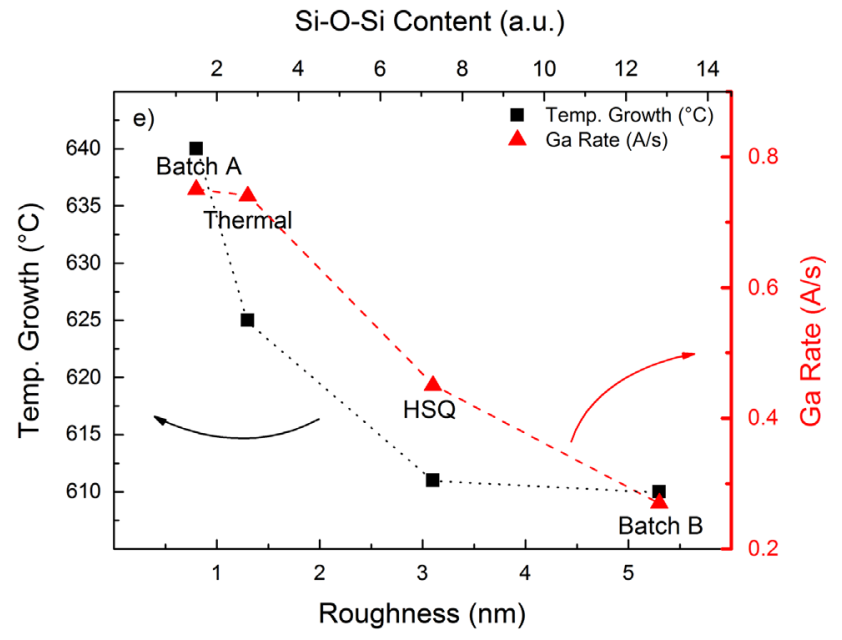

Fig. 7. The relation between optimal growth conditions (substrate temperature and $\mathrm{Ga}$ rate) at comparable density number of vertical nanowires $((1.5 \pm 0.5) \times$ $10^{5} \mathrm{~cm}^{-2}$ ) with surface roughness and interstitial oxide content is shown. The higher the $\mathrm{Si}-\mathrm{O}-\mathrm{Si}$ content, the rougher the $\mathrm{Si} / \mathrm{SiO}_{2}$-interface. To achieve comparable nanowire density lower roughness lead to higher substrate temperature and Ga rate, and vice versa.

on growth conditions has physical reason, and it is related to the interface roughness of the substrates, that seems to be correlated to $\mathrm{Si} / \mathrm{SiO}_{2}$-interface. Still, to clarify the latter point further investigation is needed. Systematic AFM and FTIR on the received substrates might help in determining the conditions to achieve the desired nanowire density. This work opens new perspectives for the reproducible integration of GaAs nanowires on silicon.

\section{Acknowledgments}

This work has been funded by ERC Starting Grant 'UpCon', ERANET-RUS InCoSiN,ITN project from FP7 'NanoEmbrace', NanoTera project 'Synergy', SNF grant nr 143908. The authors thank Martin Heiss for helpful discussions, Holger Frauenrath and Francesco Stellacci for the availability of the ATR-FTIR.

\section{Appendix A. Supplementary data}

Supplementary data associated with this article can be found in the online version at http://dx.doi.org/10.1016/j.jcrysgro.2014.07.034.

\section{References}

[1] C. Colombo, M. Heiss, M. Grätzel, A.F.i. Morral, Gallium arsenide p-i-n radial structures for photovoltaic applications, Appl. Phys. Lett. 94 (2009) 173108.

[2] M. Heiss, Y. Fontana, A. Gustafsson, G. Wüst, C. Magen, D.D. O'Regan, J.W. Luo, B. Ketterer, S. Conesa-Boj, A.V. Kuhlmann, J. Houel, E. Russo-Averchi, J.R. Morante, M. Cantoni, N. Marzari, J. Arbiol, A. Zunger, R.J. Warburton, A.F.i. Morral, Self-assembled quantum dots in a nanowire system for quantum photonics, Nat. Mater. 12 (2013) 439.

[3] P. Krogstrup, H.I. Jørgensen, M. Heiss, O. Demichel, J.V. Holm, M. Aagesen, J. Nygard, A.F.i. Morral, Single nanowire solar cells beyond the ShockleyQueisser Limit, Nat. Photon. 7 (2013) 306.

[4] C.D. Bessire, M.T. Björk, H. Schmid, A. Schenk, K.B. Reuter, H. Riel, Trap-assisted tunneling in Si-InAs nanowire heterojunction tunnel diodes, Nano Lett. 11 (2011) 4195

[5] B.j. Ohlsson, M.T. Björk, A.I. Persson, C. Thelander, L.R. Wallenberg, M. H. Magnusson, K. Deppert, L. Samuelson, Growth and characterization of GaAs and InAs nano-whiskers and InAs/GaAs heterostructures, Physica E 23 (2002) 1126.

[6] J.H. Paek, T. Nishiwaki, M. Yamaguchi, N. Sawaki, Catalyst free MBE-VLS growth of GaAs nanowires on (111)Si substrate, Phys. Status. Solidi (C) 6 (2009) 1436.

[7] F. Jabeen, V. Grillo, S. Rubini, F. Martelli, Self-catalyzed growth of GaAs nanowires on cleaved Si by molecular beam epitaxy, Nanotechnology 19 (2008) 275711.
[8] S. Breuer, C. Pfüller, T. Flissikowski, O. Brandt, H.T. Grahn, L. Geelhaar, H. Riechert, Suitability of Au- and self-assisted GaAs nanowires for optoelectronic applications, Nano Lett. 11 (2011) 1276.

[9] P. Krogstrup, R. Popovitz-Biro, E. Johnson, M.H. Madsen, J. Nygård, H. Shtrikman, Structural phase control in self-catalyzed growth of GaAs nanowires on Silicon (111), Nano Lett. 10 (2010) 4475.

[10] S. Plissard, K.A. Dick, G. Larrieu, S. Godey, A. Addad, X. Wallart, P. Caroff, Goldfree growth of GaAs nanowires on silicon: arrays and polytypism, Nanotechnology 21 (2010) 385602.

[11] S. Plissard, G. Larrieu, X. Wallart, P. Caroff, High yield of self-catalyzed GaAs nanowire arrays grown on silicon via gallium droplet positioning, Nanotechnology 22 (2011) 275602.

[12] V.G. Dubrovskii, N.V. Sibirev, Growth thermodynamics of nanowires and its application to polytypism of zinc blende III-V nanowires, Phys. Rev. B 77 (2008) 035414.

[13] E. Uccelli, J. Arbiol, C. Magen, P. Krogstrup, E. Russo-Averchi, M. Heiss, G. Mugny, F. Morier-Genoud, J. Nygård, J.R. Morante, A.F.i. Morral, Threedimensional multiple-order twinning of self-catalyzed GaAs nanowires on Si substrates, Nano Lett. 11 (2011) 3827.

[14] S.F. Fang, K. Adomi, S. Iyer, H. Morkoç, H. Zabel, C. Choi, N. Otsuka, Gallium arsenide and other compound semiconductors on silicon, J. Appl. Phys. 68 (1990) R31.

[15] E. Russo-Averchi, M. Heiss, L. Michelet, P. Krogstrup, J. Nygard, C. Magen, J.R. Morante, E. Uccelli, J. Arbiol, A.F.i. Morral, Suppression of three dimensional twinning for a $100 \%$ yield of vertical GaAs nanowires on silicon, Nanoscale 4 (2012) 1486.

[16] R.S. Wagner, W.C. Ellis, Vapor-liquid-solid mechanism of single crystal growth, Appl. Phys. Lett. 4 (1964) 89.

[17] V.G. Dubrovskii, G.E. Cirlin, V.M. Ustinov, Semiconductor nanowhiskers: synthesis, properties, and applications, Semiconductors 43 (2009) 1539.

[18] C. Colombo, D. Spirkoska, M. Frimmer, G. Abstreiter, A.F.i. Morral, Ga-assisted catalyst-free growth mechanism of GaAs nanowires by molecular beam epitaxy, Phys. Rev. B 77 (2008) 155326.

[19] M. Heiss, E. Riedlberger, D. Spirkoska, M. Bichler, G. Abstreiter, A.F.I. Morral, Growth mechanisms and optical properties of GaAs-based semiconductor microstructures by selective area epitaxy, J. Cryst Growth 310 (2008) 1049-1056.

[20] M.R. Ramdani, J.C. Harmand, F. Glas, G. Patriarche, L. Travers, Arsenic pathways in self-catalyzed growth of GaAs nanowires, Cryst. Growth Des. 13 (2013) 91-96.

[21] P. Krogstrup, H.I. Jørgensen, E. Jhonson, M.H. Madsen, C.B. Sørensen, M. Aagensen, J. Nygård, A.F.i. Morral, F. Glas, Advances in the theory of III-V nanowire growth dynamics, J. Phys. D: Appl. Phys. 46 (2013) 313001.

[22] A.F.i. Morral, C. Colombo, G. Abstreiter, J. Arbiol, J.R. Morante, Nucleation mechanism of gallium-assisted molecular beam epitaxy growth of gallium arsenide nanowires, Appl. Phys. Lett. 92 (2008) 063112.

[23] D. Rudolph, S. Hertenberger, S. Bolte, W. Paosangthong, D. Spirkoska, M. Döblinger, M. Bichler, J.J. Finley, G. Abstreiter, G. Koblmüller, Direct observation of a noncatalytic growth regime for GaAs nanowires, Nano Lett. 11 (2011) 3848

[24] Y.B. Samsonenko, G.E. Cirlin, A.I. Khrebtov, A.D. Bouravleuv, N.K. Poliakov, V.P. Ulin, V. Dubrovskii, P. Werner, Study of processes of self-catalyzed growth of GaAs crystal nanowires by molecular-beam epitaxy on modified Si (111) surfaces, Semiconductors 45 (2011) 431.

[25] U. Neuwald, H.E. Hessel, A. Feltz, U. Memmert, R.J. Behm, Initial stages of native oxide growth on hydrogen passivated $\mathrm{Si}(111)$ surfaces studied by scanning tunneling microscopy, Appl. Phys. Lett. 60 (1992) 1307.

[26] V.A. Burrows, Y.J. Chabal, G.S. Higashi, K. Raghavachari, S.B. Christman, Infrared spectroscopy of $\mathrm{Si}(111)$ surfaces after HF treatment: hydrogen termination and surface morphology, Appl. Phys. Lett. 53 (1988) 998.

[27] F.A. Soria, E.M. Patrito, P.P. Olivera, Oxidation of hydrogenated $\mathrm{Si}(111)$ by a radical propagation mechanism, J. Phys. Chem. C 116 (2012) 24607.

[28] M.P. Seah, S.J. Spencer, F. Bensebaa, I. Vickridge, H. Danzebrink, M. Krumrey T. Gross, W. Oesterle, E. Wendler, B. Rheinländer, Y. Azuma, I. Kojima, N. Suzuki, M. Suzuki, S. Tanuma, D.W. Moon, H.J. Lee, H.M. Cho, H.Y. Chen, A.T.S. Wee, T. Osipowicz, J.S. Pan, W.A. Jordaan, R. Hauert, U. Klotz, C. van der Marel, M. Verheijen, Y. Tamminga, C. Jeynes, P. Bailey, S. Biswas, U. Falke, N.V. Nguyen, D. Chandler-Horowitz, J.R. Ehrstein, D. Muller, J.A. Dura, Critical review of the current status of thickness measurements for ultrathin $\mathrm{SiO} 2$ on Si Part V: results of a CCQM pilot study, Surf. Interface Anal. 36 (2004) 1269

[29] I.W. Boyd, J.I.B. Wilson, A study of thin silicon dioxide films using infrared absorption techniques, J. Appl. Phys. 53 (1982) 4166.

[30] M. Stavola, Infrared spectrum of interstitial oxygen in silicon, Appl. Phys. Lett. 44 (1984) 514

[31] B.H. Kim, J.H. Ahn, B.T. Ahn, Finding interstitial oxygen in an Si substrate during low-temperature plasma oxidation, Appl. Phys. Lett. 82 (2003) 2682.

[32] H. Ono, T. Ikarashi, K. Ando, T. Kitano, Infrared studies of transition layers a $\mathrm{SiO}_{2} / \mathrm{Si}$ interface, J. Appl. Phys. 84 (11) (1998) 6064.

[33] K.T. Queeney, M.K. Weldon, J.P. Chang, Y.J. Chabal, A.B. Gurevich, J. Sapjeta, R.L. Opila, Infrared spectroscopic analysis of the $\mathrm{Si} / \mathrm{SiO}_{2}$ interface structure of thermally oxidized silicon, J. Appl. Phys. 87 (2000) 1322.

[34] D.A. Muller, T. Sorsch, S. Moccio, F.H. Baumann, K. Evans-Lutterodt, G. Timp, The electronic structure at the atomic scale of ultrathin gate oxides, Nature 399 (1999) 758.

[35] M. Morita, T. Ohmi, E. Hasegawa, M. Kawakami, M. Ohwada, Growth of native oxide on a silicon surface, J. Appl. Phys. 68 (1990) 1272.

[36] Y. Tu, J. Tersoff, Microscopic dynamic of silicon oxidation, Phys. Rev. Lett. 89 (2002) 086102. 
[37] M.G. Albrecht, C. Blanchette, Materials issues with thin film Hydrogen silsesquioxane low K dielectrics, J. Electrochem. Soc. 145 (1998) 4019.

[38] C.C. Yang, W.C. Chen, The structures and properties of hydrogen silsesquioxane (HSQ) films produced by thermal curing, J. Mater. Chem. 12 (2002) 1138.

[39] M. Li, Y. Hirono, S. Koukourinkova, M. Sui, Formation of Ga droplets on patterned GaAs (100) by molecular beam epitaxy, Nanoscale Res. 7 (2012) 550.

[40] T. Rieger, S. Heiderich, S. Lenk, M.I. Lepsa, D. Grützmacher, Ga-assisted MBE growth of GaAs nanowires using thin HSQ layer, J. Cryst. Growth 353 (2012) 39.

[41] X. Wang, X. Yang, W. Du, H. Ji, S. Luo, T. Yang, Thickness influence of therma oxide layers on the formation of self-catalyzed InAs nanowires on $\mathrm{Si}(111)$ by MOCVD, J. Cryst. Growth 395 (2014) 55.
[42] S.J. Gibson, J.P. Boulanger, R.R. LaPierre, Opportunities and pitfalls in patterned self-catalyzed GaAs nanowire on silicon, Semicond. Sci. Technol. 28 (2013) 105025.

[43] Y. Zhang, J. Wu, M. Aagesen, J. Holm, S. Hatch, M. Tang, S. Huo, H. Liu, Selfcatalyzed ternary core shell GaAsP nanowire arrays grown on patterned Si substrates by molecular beam epitaxy, Nano Lett. http://dx.doi.org/10.1021/ nl501565b.

[44] A.M. Munshi, D. Dheeraj, V.T. Fauske, D.C. Kim, J. Huh, J.F. Reinertsen, L. Ahtapodov, K.D. Lee, B. Heidari, A.T.J. van Helvoort, B. Fimland, H. Weman, Position-controlled uniform GaAs nanowires on silicon using nanoimprint lithography, Nano Lett. 14 (2014) 960. 